\title{
Factores Asociados al Intento Suicida e Ideación Suicida Persistente en un Centro de Atención Primaria. Bogotá, 2004-2006
}

\section{Factors associated with suicide attempts and persistent suicidal ideation at a Primary Care Unit in Bogotá, 2004-2006}

\author{
Isabel Pérez-Olmos, Milcíades Ibáñez-Pinilla, Julio C. Reyes-Figueroa, \\ Juana Y. Atuesta-Fajardo y Mónica J. Suárez-Díaz
}

Facultad de Medicina, Universidad del Rosario. Bogotá, Colombia. iperez@urosario.edu.co, galeno@cable.net.co,mippalad@yahoo.com, jcreyes_77@yahoo.com,juanis765@yahoo.com, monaye77@hotmail.com

Recibido 1 Octubre de 2007/Enviado para Modificación 1 Mayo 2008/Aceptado 7 Junio 2008

\section{RESUMEN}

Objetivos Caracterizar pacientes con intento suicida atendidos en Psiquiatría en la Unidad Primaria de Atención (UPA) La Estrella entre octubre-2004 y octubre-2005 y determinar la persistencia de ideación suicida, los factores asociados así como factores predictores.

Métodos Caracterización inicial de pacientes con intento suicida $(\mathrm{n}=156)$. Diseño posterior de casos y controles $(n=77)$ según persistencia de ideación suicida $(n=27)$. Se determinaron los factores asociados y la predicción del riesgo suicida con un modelo multivariado.

Resultados Los casos aumentaron de 6 en octubre-2004 hasta 10 en octubre-2005, con promedio mensual: $12 \pm 3,6$ casos. El $80 \%$ tenía 30 años o menor edad, 66,7 \% eran mujeres, $24,4 \%$ estudiantes y $20,5 \%$ desempleados. Como desencadenantes: disfunción familiar y conflictos de pareja en 35,2\% cada uno. El $49 \%$ consumió alcohol previo al intento suicida. La persistencia de ideación suicida se asoció con tener 31 años o más, desempleo, más de un intento suicida previo, conflictos sin resolver y disfunción familiar. La no resolución de conflictos, OR=106,7 (IC $95 \%$ : 6,8, 1668,4) y la disfunción familiar, OR=11,05 (IC95 \%: 1,7, 71,2) fueron predictores de la persistencia de ideación suicida en el modelo de regresión logística.

Conclusión La no resolución de conflictos y la disfunción familiar, predictores de la persistencia de ideación suicida en sujetos con intento suicida, el consumo de alcohol y otros factores asociados podrían controlarse con programas de prevención y promoción de salud mental.

Palabras Clave: Intento de suicidio, conflicto, relaciones familiares, factores de riesgo, Colombia (fuente: DeCS, BIREME). 


\section{ABSTRACT}

Objective Characterising patients attempting suicide who were evaluated by psychiatry at the Primary Care Unit (PCU) "La Estrella" from October/2004 to October/2005 and determining persistent suicidal ideation and associated factors.

Methods An initial description of attempted suicide patients $(n=156)$. A subsequent case control study $(n=77)$ determined persistent suicidal ideation cases $(n=27)$. A multivariate model was used for determining the remaining factors associated with persistent suicide ideation.

Results Cases increased from 6 in October/2004 to 10 in October/2005, average $12 \pm$ 3,6 cases monthly. $80 \%$ were aged 30 or younger, $66,7 \%$ were female, $24,4 \%$ were students and $20,5 \%$ were unemployed. $35,2 \%$ suffered family dysfunction and $35.2 \%$ had conflicts between couples as precipitant factors. $49 \%$ had consumed alcohol prior to the suicide attempt. Persistent suicidal ideation was associated with being 31 years old or older, being unemployed and having a higher number of previous suicide attempts, unresolved conflicts and family dysfunction. Logistical regression revealed unresolved conflicts (OR=106.7; 6,8; $1668,495 \% \mathrm{Cl}$ ) and family dysfunction (OR=11.05; 1,$7 ; 71,295 \% \mathrm{Cl}$ ) as predictor variables for persistent suicidal ideation.

Conclusion Unresolved conflicts and family dysfunction were found to be predictor factors for persistent suicidal ideation in subjects who had attempted suicide. Consuming alcohol and other associated factors should be controlled by mental health prevention and promotion programmes.

Key Words: Suicide attempted, conflict, family relationships, risk factors, Colombia (source: MeSH, NLM).

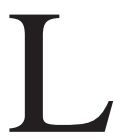
a Clasificación Internacional de Enfermedades CIE-10, define al suicidio como "la muerte ocasionada por la lesión autoinflingida informada como intencional" (1). Las expresiones de la conducta suicida incluyen entre otras: el intento suicida, definido como un acto autolesivo con cualquier grado de intención letal; el parasuicidio, o acto no mortal de autolesión deliberada; y la ideación suicida, constituida por pensamientos, planes o deseos persistentes de cometer suicidio $(2,3)$.

La prevalencia de ideación suicida en la población, según algunos autores, oscila entre el 10 y el $28 \%$; y la del intento suicida alguna vez en la vida entre el 3 y el $5 \%$ (4). Estudios internacionales presentan al intento de suicidio como el principal factor de riesgo para el suicidio consumado (4), siendo mayor el riesgo durante el primer año. La evidencia muestra que del $30 \%$ al $60 \%$ de las muertes suicidas han sido precedidas por intentos (5-7).

En Colombia, el Estudio Nacional de Salud Mental de 2003 informó que la prevalencia de intento suicida alguna vez en la vida fue 4,9\%, y de ideación 
suicida de 12,3 \% (8). En Bogotá, la tasa de suicidio por 100000 habitantes descendió de 4,7 en 2001 a 4,1 en 2002 (9); el comportamiento de la conducta suicida en Bogotá mostró una reducción en general, excepto en la Localidad de Ciudad Bolívar, donde hubo un aumento del suicidio con una tasa de 4,6 por 100 000 habitantes, mayor que la tasa de la ciudad (9).

El intento de suicidio se ha asociado a enfermedades médicas y mentales (10); a disfunción y problemas de comunicación familiar, divorcio, separación o muerte de padres o cuidadores $(10,11)$; acontecimientos negativos en la vida y otros estresores vitales ${ }^{1}$; también al antecedente de abuso físico o sexual (11$13)$, al abuso de alcohol y de sustancias psicoactivas $(2,3,5,10,14)$ y a los antecedentes familiares de suicidio consumado o de intento suicida (13). A su vez, el desempleo y el bajo nivel socioeconómico y educativo son factores de riesgo $(15,16)$.

Este estudio caracterizó el intento suicida de una población de los estratos socioeconómicos más bajos del sur de Bogotá, en la Localidad de Ciudad Bolívar, donde hay incremento de las tasas de suicidio. También, se determinaron los factores asociados a la persistencia de la ideación suicida en los mismos pacientes. Los resultados podrán orientar acciones que deben des-arrollarse para fortalecer los programas de prevención y promoción de la salud mental para controlar la persistencia del riesgo suicida.

\section{METODOLOGÍA}

La cohorte de estudio se conformó con los siguientes criterios de inclusión: pacientes de cualquier sexo y edad, valorados por el Servicio de Psiquiatría de la Unidad de Atención Primaria (UPA) La Estrella, Hospital Vista Hermosa, Localidad de Ciudad Bolívar, por intento suicida entre Octubre 1 de 2004 y Octubre 31 de 2005 que firmaron el consentimiento informado (CI) para participar en el estudio. En menores de edad se obtuvo el CI del acudiente y el asentimiento del joven. El criterio de exclusión fue la inconsistencia en la información clínica. El estudio fue aprobado por el Comité de Ética y la Dirección Científica del Hospital Vista Hermosa, UPA La Estrella. El seguimiento de la cohorte se hizo del 1 de abril al 31 de mayo de 2006 con CI verbal.

\footnotetext{
${ }^{1}$ Forero L, Cubillos D, Perez-Olmos I. Caracterización del suicidio en Bogotá durante el año 2000 y exploración de sus factores asociados. 2001. [Tesis de grado en Epidemiología]. Bogotá: TEP 212001, Hemeroteca: Biblioteca Escuela Ciencias de la Salud, Universidad del Rosario
} 
A partir de la cohorte inicial se diseñó un estudio de casos y controles; los casos fueron pacientes con persistencia de la ideación suicida, y los controles, pacientes sin ideación suicida en el seguimiento. Se encontraron 27 ca-sos y 50 controles.

Todos los pacientes consintieron en participar en el estudio. Con entrevistas individuales se recolectó la información. Para estandarizar el proceso, los investigadores diseñaron cuestionarios para cada etapa del estudio. Con el primer cuestionario se caracterizó la cohorte inicial en la valoración psiquiátrica del intento suicida. Se indagó sobre variables sociodemográficas: sexo, edad, escolaridad, estado civil, ocupación y variables del acto suicida, como el método usado y características de intentos suicidas previos. El cuestionario 2 se aplicó en entrevista telefónica en el seguimiento de la cohorte.

Para el seguimiento se llamaba al teléfono registrado. Si el paciente no se ubicaba se intentaba hablar con él en otras 2 oportunidades. Se especificó el motivo por el cual no se ubicó al paciente. Cuando se habló con él se le explicó en qué consistía el estudio, se le garantizó la privacidad y confidencialidad de la información y se obtuvo su CI verbal. A todos los pacientes se les ofreció apoyo psicoterapéutico y psiquiátrico adicional. El cuestionario 2 incluyó variables sociodemográficas y preguntas de seguimiento como persistencia de ideación suicida, ideas de muerte, el estado de ánimo predominante en ese momento de su vida, la resolución de conflictos, conflictos actuales, número de intentos suicidas previos, tipo de tratamiento recibido y la adherencia al mismo.

\section{Análisis estadístico}

En la descripción de la cohorte se usaron medidas de frecuencia absolutas y relativas para las variables cualitativas, y en las cuantitativas, medidas de tendencia central (promedio y mediana) y de dispersión (rango y desviación estándar). En el estudio de casos y controles se analizó la fuerza de asociación entre los posibles factores de riesgo y la persistencia de ideación suicida a través de la razón de disparidad (OR), y su significancia mediante el intervalo de confianza (IC) del 95 \%. También se utilizó la prueba de asociación asintótica ji-cuadrado sin corrección de Pearson, o el Test exacto de Fisher (valores esperados $<5)$ con un nivel de significancia del $5 \%(\mathrm{p}<0,05)$. Luego, se hizo el análisis multivariado mediante el OR y el IC del $95 \%$, con un modelo de regresión logística incondicional, a partir de las asociaciones bivariadas significativas o con valores de $\mathrm{p}<0,1$; se incluyeron las variables según la importancia clínica hasta obtener el modelo final, cuyo ajuste se evaluó con la prueba de HosmerLemeshow y la función de verosimilitud. Los datos se procesaron con el paquete estadístico Epi-info 6,0 y SPSS versión 15,0 para Windows. 


\section{RESULTADOS}

La cohorte se conformó con 156 individuos valorados en UPA La Estrella durante los 13 meses iniciales del estudio. En la evaluación posterior, la cohorte se redujo a 77 individuos por pérdidas del 50,6 \% (n=79); los motivos fueron: cambio de domicilio en 49 individuos, y de ciudad en 12; esto muestra una alta movilidad geográfica de esta población; otras causas fueron: prestación de servicio militar, ingreso a hogar geriátrico, un menor en Programa de Protección y una paciente que se suicidó. Esta paciente tuvo la mayoría de factores de riesgo conocidos: abuso sexual y maltrato infantil, muerte a temprana edad de la madre, cuatro intentos suicidas previos, secundaria incompleta, indigencia y pertenencia a grupos delincuenciales desde la adolescencia, farmacodependencia, disfunción familiar y conyugal y la muerte de su única hija un año y medio antes de consumar su suicidio. Recibió atención psicológica posterior a la muerte de su hija, y por los intentos suicidas tuvo atención en psiquiatría en otras instituciones. En el $4^{\circ}$ intento suicida fue tratada en UPA La Estrella con farmacoterapia y psicoterapia. Por riesgo suicida se hospitalizó en la Unidad de Salud Mental, y al egreso se mantuvo con medicación, psicoterapia individual y familiar. A pesar del seguimiento telefónico y las visitas domiciliarias no hubo adherencia terapéutica. No obstante las intervenciones realizadas, se suicidó un año después de su atención en UPA La Estrella.

Durante los 13 meses iniciales del estudio se atendió un promedio de 12 $\pm 3,6$ intentos suicidas mensuales, con un aumento progresivo en el número de casos atendidos que pasaron de 6 en octubre de 2004 a 10 casos en octubre de 2005. Las características sociodemográficas de la cohorte inicial fueron: edad promedio $24,4 \pm 10,6$ años, rango entre 8 y 67 años, género femenino, solteros, nivel educativo bachillerato incompleto o inferior y ocupación estudiante o desempleado.

El 91 \% del grupo negó tener algún antecedente personal de enfermedad médica o psiquiátrica; de consumo de sustancias en el mes anterior al intento suicida, el 55,8 \% ( $\mathrm{n}=87$ ) informó consumo de alcohol, seguido de cigarrillo con $39,1 \%(n=61)$ y otras sustancias. El 19,2 \% ( $n=30)$ informó haber sufrido abuso sexual infantil, todas ellas mujeres. El $25 \%$ (n=39) había tenido intento suicida previo y el 7,7 \% $(n=12)$ tenía antecedente familiar de intento suicida o suicidio consumado.

Respecto al método usado para realizar el intento suicida, el 89,9 \% (n=131) tuvo intoxicación exógena, seguido de lesiones con arma corto punzante en 9,6 
$\%(n=15)$ y ahorcamiento en 1,9 \% $(n=3) ; 2$ pacientes usaron lanzamiento de altura y lanzamiento hacia vehículo automotor; 5 pacientes realizaron el intento suicida usando 2 mecanismos simultáneamente. El 94,2 \% (n=147) de los intentos suicidas fue llevado a cabo en el domicilio del paciente. El 49,4 \% (n=77) refirió estar bajo los efectos del alcohol durante el intento suicida.

Los principales conflictos que los pacientes relacionaron con el intento suicida fueron: la disfunción familiar y la disfunción de pareja, cada una en 35,3 \% $(n=55)$ de la cohorte inicial, seguido por los problemas económicos en $9 \%$ ( $n=14)$, enfermedad médica o psiquiátrica, cada uno en 2,6 \% ( $n=4)$; este mismo porcentaje se encontró en problemas escolares, laborales y pérdidas afectivas recientes. El 15,4 \% (n=24) de los sujetos tuvo más de un conflicto relacionado con el intento suicida, especialmente al conjugar el aspecto económico con alguno de los otros problemas descritos. Se diagnosticó un trastorno psiquiátrico al 50,6 $\%(n=79)$ de la cohorte inicial. Dentro de estos diagnósticos, el trastorno depresivo fue el más frecuente en 78,5 \% (n=62) y el otro de la gama afectiva fue el trastorno afectivo bipolar. Otros diagnósticos psiquiátricos encontrados fueron los trastornos adaptativos, el trastorno de ansiedad, el trastorno psicótico, trastorno de personalidad límite, alcoholismo y trastorno por falta de control de impulsos, todos estos en porcentajes bastante menores.

En el seguimiento realizado a 77 pacientes de la cohorte inicial se encontró persistencia de la ideación suicida en 35,1 \% (n=27) de los pacientes. Estos últimos sujetos constituyeron los casos del diseño de casos y controles de la $2^{\mathrm{a}}$. Fase del estudio. Los factores asociados significativamente con la persistencia de ideación suicida fueron "ocupación sin función social", que incluía estar desempleado o desocupado (con exclusión de estudiantes o amas de casa); tener más de un intento suicida previo, disfunción familiar, no resolución del conflicto motivador del intento suicida, o su empeoramiento, haber requerido hospitalización en Unidad de Salud Mental, predominio de mal estado de ánimo, tener 31 o más años de edad, tener enfermedad médica, y haber requerido farmacoterapia psiquiátrica sola o combinada con psicoterapia. En la Tabla 1 se presenta la distribución de estas variables en los casos y los controles, el OR con IC del $95 \%$, y el valor de P.

En el análisis multivariado (Tabla 2) se encontró que la variable dependiente "persistencia de ideación suicida" tenía una altísima fuerza de asociación $(\mathrm{OR}=106)$ con la no resolución del conflicto motivador del intento suicida, y con menor fuerza de asociación $(\mathrm{OR}=11,05)$, aunque significativa, con la disfunción familiar, una vez controlados los efectos de las variables incluidas en 
el modelo. Otro factor importante fue la "ocupación sin función social" que mostró un OR alto $(\mathrm{OR}=5,78)$, aunque no alcanzó el nivel de significación estadística (Tabla 2).

Tabla 1. Factores asociados a la persistencia de ideación suicida, UPA La Estrella, Ciudad Bolívar, 2006, Bogotá

\begin{tabular}{|c|c|c|c|c|}
\hline Variables & $\begin{array}{l}\text { Casos } \\
\mathrm{N}(\%)\end{array}$ & $\begin{array}{l}\text { Controles } \\
\mathrm{N}(\%)\end{array}$ & $\mathbf{P}$ & OR (IC95\%) \\
\hline Familiar con intento o suicidio & $5(18,5)$ & $3(6,0)$ & 0,095 & $3,56(0,66-21,05)$ \\
\hline $\begin{array}{l}\text { Número de intentos } \\
\text { Previos } \geq 2\left({ }^{* *}\right)\end{array}$ & $13(48,1)$ & $7(14,0)$ & 0,001 & $5,7(1,68-19,99)$ \\
\hline Estado de ánimo Reg-mal ("*) & $27(100)$ & $14(28,0)$ & $<0,001$ & \\
\hline Ideas de muerte ("*) & $24(88,9)$ & $4(8,0)$ & $<0,001$ & $92(19,022-444)$ \\
\hline Disfunción Familiar ("*) & $19(50)$ & $19(50)$ & 0,0067 & $3,875(1,42-10,57)$ \\
\hline Disfunción de pareja & $10(37,0)$ & $17(34,0)$ & 0,791 & $1,14(0,38-3,38)$ \\
\hline Económico & $8(29,6)$ & $13(26,0)$ & 0,733 & $1,20(0,37-3,81)$ \\
\hline Enfermedad médica (*) & $3(11,1)$ & 0 & $0,040(f)$ & \\
\hline Enfermedad psiquiátrica & $2(7,4)$ & 0 & $0,120(f)$ & \\
\hline Otras enfermedades & $1(3,7)$ & $2(4,0)$ & $0,720(f)$ & \\
\hline Conflicto y resolución ("*) & $26(96,3)$ & $14(28,0)$ & $<0,001$ (f) & f) $66,86(8,16-1452,14)$ \\
\hline $\begin{array}{l}\text { Hospitalización Unidad Salud } \\
\text { Mental ("*) }\end{array}$ & $13(48,1)$ & $7(14,0)$ & 0,001 & $5,70(1,9-17,12)$ \\
\hline Psicofarmacoterapia ("*) & $14(51,9)$ & $10(20,0)$ & 0,004 & $4,31(1,55-12,00)$ \\
\hline Psicoterapia & $9(33,3)$ & $8(16,0)$ & 0,08 & $2,63(0,87-7,89)$ \\
\hline Psicoterapia/farmacoterapia & $6(22,2)$ & $2(4,0)$ & 0,012 & $6,86(1,28-36,81)$ \\
\hline
\end{tabular}

El porcentaje de predicción del modelo multivariado fue de 88,3 \% con un buen ajuste del modelo logístico para explicar la persistencia de la ideación suicida con las variables incluidas como indican los resultados de la prueba de bondad de ajuste (prueba de $\mathrm{X}^{2}$ de Hosmer-Lemeshow=3,718, $\mathrm{p}=0,882$ )

\section{DISCUSIÓN}

La mayoría de la literatura existente sobre el fenómeno suicida se refiere principalmente a la caracterización del suicidio consumado $(3,7,9,16)$. No se encontraron artículos sobre la persistencia de la ideación suicida después de realizado un intento suicida. Este estudio mostró que las características de las personas que realizaron intentos suicidas coinciden con las presentadas en la literatura científica como son: predominio en población joven (5,10,16-18), sexo "femenino", y "soltero" como categoría del estado civil $(2-5,12,14)$. Esto último muestra la importancia de la estabilidad del vínculo afectivo y del apoyo sociofamiliar como factores protectores en la depresión y en el suicidio. La ocupación con más alto porcentaje fue la de "estudiante", lo cual es acorde con la población 
joven descrita en el estudio cuya edad promedio fue 24,4 \pm 10,6 años. El "desempleo" ocupa los primeros lugares en las estadísticas de factores relacionados con suicidio $(5,9,12,13)$.

Tabla 2. Modelo de Regresión Logística de persistencia de ideación suicida, UPA La Estrella, Ciudad Bolívar, 2006, Bogotá

\begin{tabular}{|c|c|c|c|c|c|c|}
\hline \multirow[t]{2}{*}{ Factores asociados } & \multirow[t]{2}{*}{ B } & \multirow{2}{*}{$\begin{array}{l}\text { Error } \\
\text { est. }\end{array}$} & \multirow[t]{2}{*}{$\mathrm{P}$} & \multirow[t]{2}{*}{ OR } & \multicolumn{2}{|c|}{ IC $95 \%$ OR } \\
\hline & & & & & Inferior & Superior \\
\hline Edad $\geq 31$ & 1,247 & 1 & 0.212 & 3,48 & 0,49 & 24,707 \\
\hline Ocupación Sin función social & 1,756 & 1,397 & 0,209 & 5,788 & 0,375 & 89,405 \\
\hline Vive solo & 0,205 & 0,372 & 0,581 & 1,228 & 0,592 & 2,546 \\
\hline Sin resolución de conflictos & 4,671 & 1,402 & 0,001 & 106,79 & 6,836 & 1668,439 \\
\hline Dos o más intentos prévios & 1,236 & 1,007 & 0,22 & 3,441 & 0,479 & 24,743 \\
\hline Disfunción familiar & 2,403 & 0,95 & 0,011 & 11,052 & 1,715 & 71,202 \\
\hline Farmacoterapla & 1,248 & 1,113 & 0,262 & 3,483 & 0,393 & 30,844 \\
\hline Psicoterapia & 0,547 & 1,545 & 0,723 & 1,728 & 0,084 & 35,728 \\
\hline Psicoterapia y farmacoterapia & 1,07 & 2,238 & 0,663 & 2,915 & 0,036 & 234,33 \\
\hline Constante & $-7,819$ & 2,43 & 0,001 & & & \\
\hline
\end{tabular}

La frecuencia de la intoxicación exógena como método suicida fue similar al de la literatura, y es el método predominante en el intento suicida, especialmente en nuestro medio (18), seguido de las heridas con arma blanca. El método podría depender de la disponibilidad del mismo y de los recursos económicos y psicológicos del sujeto $(2,3,14)$; la baja frecuencia del uso de arma de fuego podría estar relacionado con baja accesibilidad en este grupo poblacional y con las campañas de desarme en la ciudad.

El alcohol fue la principal sustancia asociada al intento suicida, tiene aceptación y gran permisividad social en la cultura local, y además, favorece la impulsividad, compromete la capacidad autocrítica y agrava los estados depresivos y las comorbilidades psiquiátricas del sujeto $(2,4,5,10,14,18)$.

El antecedente de abuso sexual, enfermedad médica o mental, como factores de riesgo para intento suicida han sido referidos en otros estudios $(5,10,13,14)$; sin embargo, aunque se encontraron frecuencias importantes, pues la quinta parte de las personas evaluadas, todas ellas mujeres, tuvo el antecedente de abuso sexual, esta variable no alcanzó a tener asociación significativa con la persistencia de la ideación suicida, lo mismo ocurrió con la presencia de una enfermedad psiquiátrica, quizá por el tamaño de la muestra en la $2^{\text {a }}$ fase del estudio. Los diagnósticos psiquiátricos principales encontrados fueron los trastornos depresivos, también concordante con la literatura, seguido por trastornos adaptativos y trastorno límite de la personalidad (3,5,13,14,16-19). 
La persistencia de ideación suicida en 35,1 \% de los 77 pacientes entrevistados en la $2^{\mathrm{a}}$ fase del estudio no pudo ser comparada con datos de la literatura científica pues no se encontraron estudios de seguimiento de la ideación suicida luego de la atención psiquiátrica por intento suicida.

La persistencia de ideación suicida fue más frecuente de manera estadísticamente significativa entre quienes tenían 31 años o más, no tenían ocupación con función social, tenían conflictos no resueltos o empeorados, disfunción familiar, una enfermedad médica, un mayor número de intentos suicidas, habían recibido farmacoterapia psiquiátrica con o sin psicoterapia, y habían estado hospitalizados en Unidad de Salud Mental; todas estas características podrían mostrar un perfil de la severidad de la disfunción psiquiátrica crónica y en la dimensión familiar y social. Los pacientes hospitalizados en la Unidad de Salud Mental fueron justamente los que durante la entrevista manifestaron persistencia de la ideación suicida, tenían un trastorno depresivo grave o una patología psiquiátrica activa, tenían una red de apoyo débil o un estado de conflicto no resuelto, o realizaron un intento suicida de alta letalidad. A su vez, a estos mismos individuos son a quienes se les prescribe más farmacoterapia psiquiátrica, sola o combinada con psicoterapia, debido a la mayor gravedad del trastorno psiquiátrico que presentan como se muestra en la Tabla 1.

Estas variables podrían indicar de manera indirecta una red de apoyo social inexistente o débil y carencia de recursos para la resolución de conflictos, junto a bajas coberturas y baja efectividad de los programas de promoción, prevención e intervención en Salud Mental Comunitaria en la localidad de referencia $(3,4,16)$.

El modelo de regresión logística mostró que la variable dependiente "persistencia de ideación suicida" fue mejor explicada por la "no resolución del conflicto" y la "disfunción familiar", una vez controlado el efecto de las demás variables incluidas en el modelo. En la literatura revisada no se encontraron artículos que plantearan hallazgos similares.

Los resultados de este estudio corroboran el hecho de que la conducta suicida es un fenómeno polimórfico, influenciado por múltiples factores que condicionan su desarrollo, perpetuación y desenlace. La determinación de estos factores junto a la pronta y efectiva intervención de los factores de riesgo controlables favorece la interrupción de un proceso que de otra manera tiene resultados funestos para el individuo, la familia y sociedad. 
Limitaciones del estudio

Las pérdidas de sujetos valorados por intento suicida en UPA La Estrella durante los 13 meses iniciales del estudio fueron cercanas a la mitad de la cohorte inicialmente constituida, éstas pérdidas constituyen una limitación del estudio pues plantea la duda acerca de si los resultados del diseño de casos y controles realizado en la $2^{\text {a }}$ fase del estudio se mantendrían, se incrementarían o disminuirían si se hubiese podido obtener los datos de toda la cohorte inicial o de la mayoría de ella. El control de este probable sesgo se hizo mediante la comparación de las variables de la cohorte inicial entre los dos grupos (casos y controles y grupo de pérdidas) sin encontrar diferencias significativas. Los factores de riesgo identificados en este estudio concuerdan con la literatura sobre tentativas de suicidio y patrones socioculturales de poblaciones colombianas vulnerables (18), lo que también apoyaría la probabilidad de que estas asociaciones se habrían mantenido con los datos de la cohorte completa.

\section{Conclusiones}

Este estudio realizado con una población vulnerable de la capital de Colombia, mostró que los intentos suicidas se presentaron predominantemente en población joven, de sexo femenino, con familias disfuncionales, conflictos precipitantes del suicidio no resueltos, antecedentes de maltrato infantil, trastornos depresivos, de adaptación y enfermedad médica, y con intento suicida realizado bajo efectos de alcohol.

La alta frecuencia de la conducta suicida observada en la Localidad Ciudad Bolívar y de los factores que incrementan la probabilidad de su presentación y de la persistencia de la ideación suicida podrían ser reflejo de la situación crítica que se vive en esa zona marginal de Bogotá, cuya población tiende al intento suicida o al suicidio consumado, como la única opción de solución o por lo menos de escape y alivio a la tensión y sufrimiento que viven.

Los factores asociados a la persistencia de la ideación suicida luego del intento suicida fueron: tener 31 o más años de edad, ser estudiante o estar desempleado o desocupado, tener más de dos intentos suicidas previos, la no resolución o el empeoramiento de los conflictos desencadenantes del intento suicida para el que recibieron atención psiquiátrica, y la disfunción familiar. Estas dos últimas variables podrían predecir la perpetuación del riesgo suicida en el grupo de estudio. Por lo tanto, se requiere con urgencia una reestructuración de los programas de promoción y prevención en salud mental con un abordaje 
multidisciplinario con enfoque bio-psico-social dirigido a la interrupción del proceso hacia el suicidio •

Agradecimientos. A los pacientes y familias, así como al personal de UPA La Estrella, Hospital Vista Hermosa, en la Localidad Ciudad Bolívar de Bogotá por la colaboración para la realización de este estudio.

\section{REFERENCIAS}

1. Organización Mundial de la Salud (OMS). Clasificación Internacional de Enfermedades CIE-10. Madrid: Organización Mundial de la Salud; 1992.

2. Instituto Nacional de Medicina Legal y Ciencia Forenses, Centro de Referencia Nacional sobre Violencia. Ideación, Intento y Suicidio en Bogotá. Un estado del Arte. Bogotá, DC: Panamericana Formas e Impresos SA; 2003.

3. Alcaldía Mayor de Bogotá, Secretaria de Gobierno. Investigación sobre el suicidio en Bogotá. Facultad de Ingeniería, Facultad de Ciencias Humanas, Centro de Estudios Sociales. Bogotá: Universidad Nacional de Colombia; 2001.

4. Botega N. Suicidal behaviour in the community: prevalence and factors associated with suicidal ideation. Rev Bras Psiquiatr 2005; 27(1):45-53.

5. Gómez-Restrepo C, Rodríguez N, Bohórquez A, Díaz granados N, Ospina M y Fernández C. Factores asociados al intento suicida en la población colombiana. Rev Col de Psiquiatría 2002; 31(4): 271-286.

6. Moscicki EK. Identification of suicide risk factors using epidemiologic studies. Psychiatr Clin North Am 1999; 20(3): 499-517.

7. Palacio-Acosta C, García-Valencia J, Diago-García J, Zapata C, Ortiz-Tobón J, et al. Characteristics of people committing suicide in Medellín, Colombia. Rev Salud Pública (Bogotá) 2005;7(3):243-253.

8. Ministerio de Protección Social. Informe preliminar, Estudio Nacional de Salud Mental y estilos de vida 2003. [internet]. Disponible en: www.ministerioproteccionsocial.gov.co. Consultado mayo 2004

9. Veeduría Distrital de Bogotá. Muertes violentas en Bogotá 1993-2002. Vivir en Bogotá, Boletín No. 10;2003:12-21. [Internet]. Disponible en: www.veeduriadistrital.gov.co. Consultado en mayo 2004.

10. Qin P, Agerbo E. Suicide risk in relation to socioeconomic, demographic, psychiatric and familial factors: A national register based study of all suicides in Denmark 1981-1997. Am J Psychiatry 2003;160(4):765-772.

11. Agerbo E, Nordentoft M, Mortensen P. Familial, psychiatric, and socioeconomic risk factors for suicide in young people: Tested case-control study. Br Med J 2002; 13: 325-355.

12. Calvo J, Sanchez R, Tejada P. Prevalencia y Factores asociados a ideación suicida en estudiantes universitarios. Rev. Salud Pública (Bogotá) 2003;5(2):123-143

13. Heikkinen M, Isometsa ET, Marttunen MJ, Aro HM, Lonnqvist JK. Social factors in suicide. B J Psychiatry $1995 ; 167: 747-753$.

14. Organización Mundial de la Salud (OMS). Trastornos mentales y cerebrales. Departamento de salud mental y toxicomanías. Ginebra (Suiza) : Organización Mundial de la Salud; 2001.

15. Youssef G. Personality trait risk factors for attempted suicide among young women with eating disorders. Eur Psychiatry 2004; 19 (3): 131-139.

16. Sánchez R, Orejarena S, Guzmán Y. Características de los suicidas en Bogotá: 1985-2000. Rev. Salud Pública (Bogotá) 2004;6(3):217-234. 
17. Miller A, Glinski J. Youth suicidal behaviour: Assessment and intervention. J clin psychol 2000; 56(9):1.131-1.152.

18. Pérez-Olmos I, Rodríguez-Sandoval E, Dussán-Buitrago MM, Ayala-Aguilera JP. Caracterización psiquiatrica y social del intento suicida atendido en una clínica infantil, 2003-2005. Rev. Salud Pública (Bogotá) 2007;9(2):230-240.

19. Hansen P, Wang A, Stage K. Comorbid personality disorder predicts suicide after major depression: a 10 year follow-up. Act Psychiatr Scand 2003;107(6):436-440. 